\title{
Effects of treatment of temporomandibular disorders on headache, quality of life, and neck function in patients with tension-type headaches: a randomized controlled study
}

\author{
Wonjae Choi ${ }^{a(i)}$, Jungmuk Woo ${ }^{b(1)}$, Sangbong Lee ${ }^{a, b(1)}$, Seungwon Lee ${ }^{a, c(1)}$ \\ anstitute of SMART Rehabilitation, Sahmyook University, Seoul, Republic of Korea \\ ${ }^{b}$ Department of Physical Therapy, The Graduate School, Sahmyook University, Seoul, Republic of Korea \\ 'Department of Physical Therapy, College of Health Science and Social Welfare, Sahmyook University, Seoul, Republic of Korea
}

\begin{abstract}
Objective: Tension-type headaches usually occur with temporomandibular disorder, which increases the risk of the chronic tension-type headaches. This study was conducted to investigate the effect of additional temporalmandibular therapy compared to cerivcal joint therapy for tension-type headaches with pericranial tenderness.
\end{abstract}

Design: Randomized controlled trial.

Methods: Forty-one patients with chronic tension-type headaches and pericranial tenderness were randomized into the 3 groups, such as the temporomandibular joint therapy group (TMJT group, $n=14)$, cervical manual therapy group (CMT group, $n=14)$ and conservative therapy group (CT group, $n=13$ ). All patients were assessed at baseline and after each intervention during the three sessions. The participants in the TMJT group received the temporomadibular joint treatment and cervical manual therapy for 30 minutes, once a week, for 3 weeks. The participants in the CMT group received the cervical manual therapy, and those in the CT group received modalities during same time period. The outcome measurements used were the intensity of headaches measured on the Visual Analogue Scale (VAS), quality of life measured with the Headache Impact Test (HIT-6), and function of the cervical spine using the Neck Diability Index (NDI).

Results: The TMJT group that received temporomandibular joint treatment and cervical manual therapy showed a significant decrease in VAS, HIT-6, and NDI compared with the other 2 groups $(p<0.05)$.

Conclusions: This study suggected that temporomandibular joint treatment combined with cervical manual therapy was more effective for the chronic tension-type headaches with pericranial tenderness than the usual cervical therapy alone.

Key Words: Neck, Quality of life, Temporomandibular joint, Tension-type headache, Visual analog scale

\section{Introduction}

Headache is the most common form of pain that everyone experiences at least once in their lifetime [1]. The World Health Organization defined headaches as one of the top ten causes of harm to human health [2], and in the United States, the social losses due to headache was estimated at 1 billion dollars annually [3]. When there is no specific cause and on- ly headache symptoms appear, it is called a primary headache, and headaches that occur with other diseases are called a secondary headache [4]. The most common type of a primary headache is a tension headache [5].

Tension headaches are caused by stress, overwork, tension, etc., which causes constant muscle contraction in the occipitocervical area and causes pain, and refers to pain that spreads throughout from the occiput to the parietal [6].

Received: 1 December, 2020 Revised: 23 December, 2020 Accepted: 24 December, 2020

Corresponding author: Seungwon Lee (ORCID https://orcid.org/0000-0002-0413-0510)

Institute of SMART Rehabilitation, Sahmyook University, 815 Hwarang-ro, Nowon-gu, Seoul 01795, Republic of Korea

Tel: 82-2-3399-1630 Fax: 82-2-3399-1639 E-mail: swlee@syu.ac.kr

(c) This is an Open-Access article distributed under the terms of the Creative Commons Attribution Non-Commercial License (http://creativecommons.org/licenses/ by-nc/4.0) which permits unrestricted non-commercial use, distribution, and reproduction in any medium, provided the original work is properly cited.

Copyright $@ 2020$ Korean Academy of Physical Therapy Rehabilitation Science 
Symptoms cause not only pain, but also various dysfunctions such as decreased range of motion of the cervical vertebra, sleep disturbance, and decreased concentration [7]. Chronic tension headaches appear for more than 6 months and more than 15 days a month, appear bilateral, and the pain does not worsen through daily life [8]. Those with chronic tension headaches often complain of pain only in the pericranial region, but they often complain of trismus, pain in the temporomandibular joint, and crepitation [9]. According to previous studies, about $70 \%$ of patients with temporomandibular joint complained of a headache [10], and compared with those without a headache, those with temporomandibular joint dysfunction complained more [11]. Temporomandibular joint dysfunction has been reported to form several types of headache, such as myofascial, tensional, and cervicogenic headaches around the temporal and mandibular joint pathophysiologically $[12,13]$.

Therefore, temporomandibular joint treatment is one of the factors to be considered in the treatment of patients with tension headaches. Although the relationship between temporomandibular joint disorders and tension headaches is still unclear [14], it was reported that treatment of the temporomandibular joint had a significant effect on headache relief in patients with cervical vertebral headache related to pericranial tenderness $[15,16]$.

In the study of Saha et al. [17], occlusal splint therapy was performed on patients with migraine and tension headaches, but there was no significant difference from the group to which drugs or general treatment were applied.

Therefore, there are insufficient studies on the treatment of the temporomandibular joint in patients with chronic tension headaches, and no studies have applied direct intervention to the muscles involved in the movement of the temporomandibular joint. Therefore, the purpose of this study was to investigate the effects of temporomandibular joint treatment in patients with tension headaches on headache, quality of life, and neck function.

\section{Methods}

\section{Participants and procedure}

This study was designed as a randomized trial, and among the patients with tension headache who were being treated in orthopedic surgery, those who understood this study and wished to participate were recruited. Consent was obtained after explaining the procedure and purpose of the study to all subjects, and the study was approved by the Research Ethics
Committee of Sahmyook University (2016069HR).

The selected subjects were those who complained of tension headaches and were diagnosed as chronic tension headache patients related to pericranial tenderness classified in the International Headache Disease Classification, those who had headache symptoms after 6 months, and those who had tenderness in the masseter muscle. On the other hand, those who received treatment related to this study in the last 1 year, who received injection therapy or surgical operation, and patients with severe disc herniation or fracture were excluded.

Thirty-three patients with tension headaches related to pericranial tenderness were recruited, and 8 patients meeting the exclusion criteria were excluded. Forty-five patients were evaluated for headache, quality of life, and neck function through pre-tests. Participants were randomly divided into 15 patients in the temporomandibular joint treatment group, 15 patients in the cervical manual therapy group, and 15 patients in the conservative water treatment group. In the same manner, each intervention was applied once a week for 30 minutes for 3 weeks. In the temporomandibular joint group, cervical manual therapy and temporomandibular joint therapy were applied for 30 minutes, in the cervical manual therapy group, only cervical manual therapy was applied for 30 minutes, and in the conservative water treatment group, percutaneous nerve stimulation therapy, ultrasound, and warm compress were applied for 30 minutes. Post-measurement was performed every week of treatment to determine the effect of the intervention.

\section{Intervention}

\section{Temporomandibular joint treatment}

Temporomandibular joint treatment was performed in the temporomandibular joint treatment group with cervical manual therapy for 30 minutes. Temporomandibular joint treatment was applied by modifying the intervention performed in the study by Von Piekartz [16]. The goal of treatment is to induce pain in the chewing muscle and to relax the shortened muscle, and to treat the limitation of the range of motion of the temporal mandibular joint [18]. The muscles that received treatment were the frontalis, temporalis, masseter, and the medial pterygoid. Specific approaches included deep tendon friction massage of the muscles that indicated pain inside and outside the oral cavity, and direct caudal, ventrocaudal, ventral, and mediolateral displacement of the temporomandibular joint operation.

The basic principle of temporomandibular joint treatment 
is that smooth movement along the curved surface occurs in the condyle, and the disc of the temporal mandibular joint should be well positioned in front of the joint ridge [19].

In addition, the temporomandibular joint is capable of lateral sliding and excursion sliding, so deviations from the joint must be addressed.

\section{Cervical manual therapy}

To the cervical manual therapy group, manual therapy of the cervical joint was applied for 30 minutes. As for the treatment method, the method of the preceding paper was modified and applied according to this study, and includes soft tissue access [20], joint movement, and manual correction [21]. For the soft tissue approach, deep tendon friction massage and ischemic compression were performed on the sternocleidomastoid, splenius capitis and cervicis, trapezius, scalenes, and levator scapulae, and shoulder lifters. Fascial relaxation is applied to the soft tissue under the larynx that causes impairment in the movement of the atlantooccipital joint. The suboccipital muscles are the most common causes of tension headaches. In this treatment, the patient relaxed the laryngeal region over the therapist's hand in a supine position, and the therapist used the fingers to relax and stretch the suboccipital muscles. A deep, increasing pressure was applied horizontally to the tissues. The duration of the treatment was 10 minutes, and the patient closed his or her eyes and breathed comfortably. In the articulation approach, a joint movable technique was applied in which the patient complained of the most pain among the segments of the cervical joint in the prone position, from the rear of the spinous process to the front, and a more severe pain among the left and right transverse processes was applied. If one of the left or right transverse processes elicited more severe pain, joint mobilization that pushed from the transverse process to the posterior-anterior was applied.

\section{Conservative physical therapy}

For the conservative physical therapy group, percutaneous stimulation therapy, ultrasound therapy, and warm compress were applied for 30 minutes.

The warm compress was applied to the periphery of the cervical vertebra for 10 minutes, and then percutaneous electrical nerve stimulation treatment was applied to the splenius capitis and cervicis, trapezius, the scalenes, and the levator scapula for 15 minutes. Ultrasound treatment was applied to the periphery of the temporal and mandibular joints for 5 minutes by using a $1.5 \mathrm{MHz}$ ceramic and con- tinuous ultrasound with an intensity of $1.5 \mathrm{Watt} / \mathrm{cm}^{2}$.

\section{Outcome measurements}

A Visual Analogue Scale was used to measure the pain levels related to the headaches. This tool is used to assess the pain that the patient reports subjectively, and it is $100 \mathrm{~mm}$ long. The outer side is $0 \mathrm{~mm}$, which means there is no pain, and the right side is $100 \mathrm{~mm}$ and indicates the greatest pain that the patient can feel [22]. In this study, the patient's current headache pain level was directly marked on an examination paper, and the marked part was measured and recorded with a ruler. The patient was prevented from retesting errors by not knowing the previously evaluated values during retesting. The reliability of the evaluation tool was 0.97 , the intra-measure reliability was 1.00 , and the inter-measure reliability was 0.99 , which was very high [23].

To evaluate the quality of life, the Headache Impact Test-6 (HIT-6) was used. This tool was developed to investigate the effect of the overall headache, including migraine and tension headache, on the patient's overall life. A total of 6 questions were asked to measure pain, social function, role function, cognitive function, psychological pain, and vitality. A score of 49 or less was classified as a case with no or insignificant effect of a headache, a case of 50 to 55 was a slight effect of a headache, a score of 56 to 60 was classified as significantly affected, and a score above 60 was a case of a severe effect of a headache [24]. The internal consistency (Cronbach's alpha) of the headache effect test was 0.89 , and the reliability of the retest was 0.80 , showing high reliability [25].

\section{The Neck Disability Index (NDI) was used to evaluate neck function}

This tool is a questionnaire to find out how much of the pain and discomfort of the cervical vertebra causes disorders in daily life, and a total of 10 items are included: intensity of pain, self-care, lifting, reading, headache, concentration, work, driving. It consists of life span and filtration activity. If the score exceededs 34 points, it is interpreted as complete, 25 to 35 severe, 15 to 24 moderate, 5 to 14 mild, and 0 to 4 none [26]. Complete means the least functional, and the lower the score, the better. In this study, 0 points were converted to $100 \%$ and was calculated. The internal consistency of the NDI (Cronbach's alpha) is 0.88 , showing high reliability [27]. 


\section{Data analysis}

All work and statistical analysis was performed using IBM SPSS Statistics for Windows, Version 19.0 (IBM co., Chicago, IL, USA). Normality test by Kolmogorov-Smirnov was performed, and chi-square test and one-way ANOVA were used to evaluate the prior homogeneity of each group. Two-way repeated measures ANOVA was used to analyze the difference between the 3 groups between repeated measurement periods, and then least significant difference (LSD) was used for post-test analysis. The statistical significance level of the data was set to 0.05 .

\section{Results}

A total of 45 subjects who met the selection criteria participated in the study, but 4 dropped out, leaving a remainder of 41 subjects for analysis.

All variables of the subjects were normally distributed, and there was no significant difference between the general characteristics of the three groups $(p>0.05)$ (Table 1$)$.

All dependent variables were interpreted as a univariate test by establishing the Mauchy's sphericity test assumption.

Changes in headache, quality of life, and neck function among the 3 groups are presented in Figure 1. In the univariate test results, the interaction between the time and groups was significant $(p<0.05)$. In the intra-individual effect test, the interaction between the time and group was significant $(p<0.05)$. To compare the differences between groups, the LSD analysis showed a significant effect in the temporomandibular joint treatment group $(p<0.05)$. The temporomandibular joint treatment group decreased by $3.21 \mathrm{~cm}$ after the $1 \mathrm{st}$ treatment, $1.14 \mathrm{~cm}$ after the $2 \mathrm{nd}$ treatment, and $1.75 \mathrm{~cm}$ after the $3 \mathrm{rd}$ treatment, and there was a statistically

Table 1. General characteristics of participants

$(\mathrm{N}=41)$

\begin{tabular}{lcccc}
\hline \multicolumn{1}{c}{ Characteristics } & TMJT group $(\mathrm{n}=14)$ & CMT group $(\mathrm{n}=14)$ & CT group $(\mathrm{n}=13)$ & $p$ \\
\hline Sex (male/female) & $7 / 7$ & $8 / 6$ & $8 / 5$ & 0.840 \\
Age $(\mathrm{y})$ & $35.00(11.82)$ & $34.71(7.50)$ & $42.62(11.00)$ & 0.092 \\
Height $(\mathrm{cm})$ & $166.36(8.39)$ & $169.57(7.52)$ & $167.46(8.23)$ & 0.568 \\
Weight $(\mathrm{kg})$ & $63.57(7.79)$ & $67.57(12.06)$ & $66.69(7.98)$ & 0.513 \\
\hline
\end{tabular}

Values are presented as number only or mean (SD).

TMJT: temporomandibular joint therapy, CMT: cervical manual therapy, CT: conservative therapy.
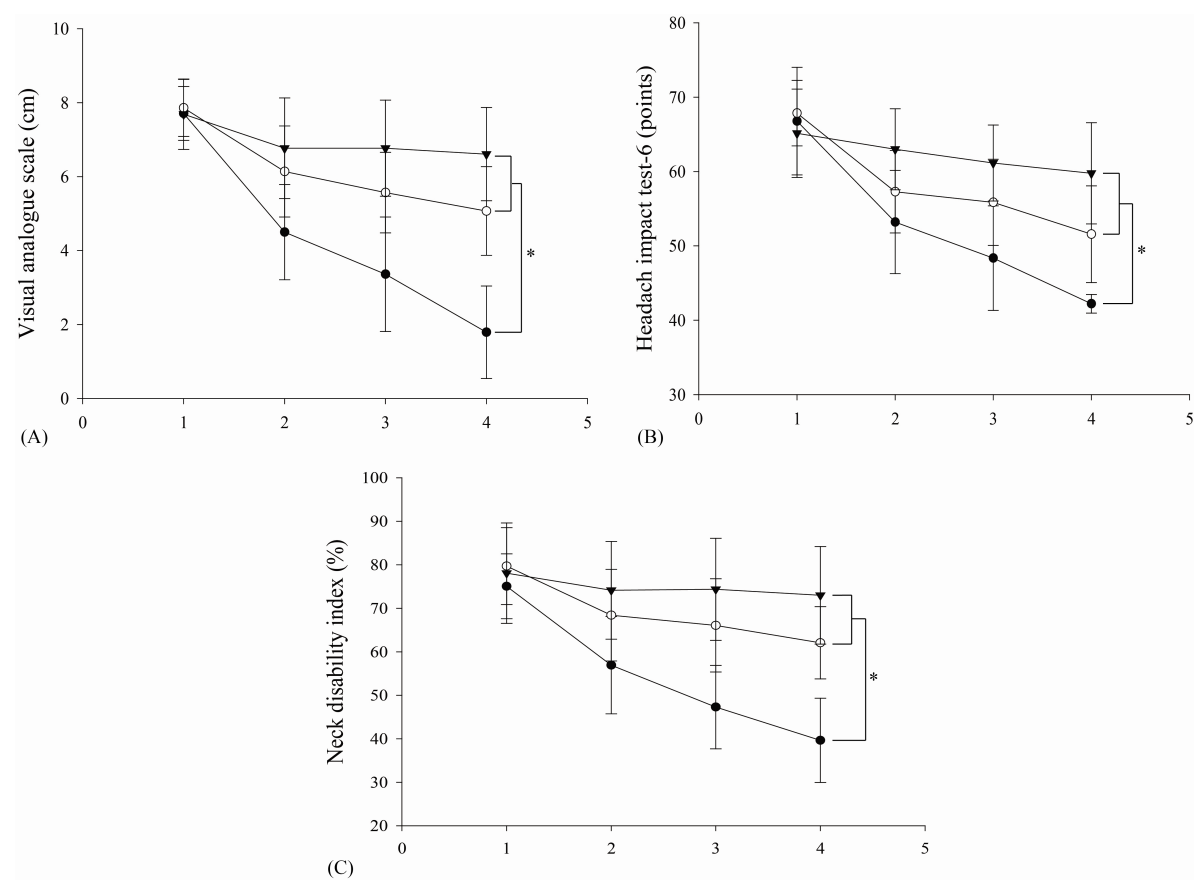

Figure 1. The black circle represents the TMJT group and the white circle is CMT group, and black triangle is $\mathrm{CT}$ group. (A) Intensity of headache, (B) quality of life, (C) neck function. TMJT: temporomandibular joint therapy, CMT: cervical manual therapy, CT: conservative therapy. ${ }^{*} p<0.05$. 
significant difference $(p<0.05)$. In the results of the univariate test for quality of life, the interaction between time and group was found to be significant $(p<0.05)$. In the results of the intra-individual effect test, the interaction between the time and groups was also significant $(p<0.05)$. The LSD test was performed to compare the differences between groups, and as a result, the effect was most significant in the temporomandibular joint treatment group $(p<0.05)$. The temporomandibular joint treatment group decreased by 13.58 points after the 1 st treatment, 4.85 points after the 2 nd treatment, and 6.15 points after the 3 rd treatment, and there was a statistically significant difference $(p<0.05)$.

The univariate test for neck function showed significant interaction between time and groups $(p<0.05)$. In the result table of the intra-individual effect test, there was also an interaction between the time and group $(p<0.05)$. LSD test was performed to find out the difference between the groups, and there was a significant difference in temporal mandibular joint compared to the other 2 groups $(p<0.05)$. The temporomandibular joint treatment group decreased to $18.14 \%$ after the 1st treatment, $9.26 \%$ after the 2nd treatment, and $7.65 \%$ after the 3 rd treatment, and there was a statistically significant difference $(p<0.05)$.

\section{Discussion}

In this study, the effects of temporomandibular joint treatment and cervical manual therapy on pain, quality of life, and neck function in patients with chronic tension headache were more significant than those of the other 2 groups in the temporomandibular joint treatment group, indicating a significant difference $(p<0.05)$.

In a previous study, the changes in pain intensity in patients with tension headaches after manual treatment at the cervical joint and the surrounding area was tested, and manual therapy was performed on the cervical joint. After 8 weeks of treatment, the intensity of headache pain decreased by $78 \%$, and after 26 weeks it was also decreased by $73 \%$ [28]. In another previous study, 82 patients with tension headaches were divided into an experimental group to which cervical manual therapy was applied, and a control group to which conservative physical therapy was applied to study the intensity, frequency, and duration of the headache [29]. As a result, the headache intensity decreased by $43 \%$ in the experimental group and by $16 \%$ in the control group $(p<0.001)$, and the frequency of headache decreased by $77 \%$ in the experimental group and by $44 \%$ in the control group, showing a significant difference between the 2 groups $(p<0.001)$. The duration of the headache decreased by $46 \%$ in the experimental group and by $16 \%$ in the control group $(p<0.001)$. As a result of this study, there were significant differences before and after intervention in all groups $(p<0.05)$, but the temporomandibular joint treatment group showed a more significant improvement compared to the other 2 groups $(p<0.05)$.

The muscles used for the diagnosis of tension headaches related to cranial tenderness are the frontalis, temporalis, masseter, pterygoid, sternocleidomastoid, splenius capitis and cervicis, and the trapezius [4]. The sternocleidomastoid, splenius capitis and cervicis, and trapezius muscles are directly involved in the stability of the cervical vertebra, and the temporalis, masster and the pterygoid muscles are chewing muscles that are directly involved in the movement of the temporomandibular joint [30]. Due to the structural association of these muscles, patients with tension headaches related to pericranial tenderness share problems with the temporomandibular joint, cervical joint and the surrounding muscles [31]. Therefore, in the temporomandibular joint treatment group, when the cervical joint treatment and the temporomandibular joint treatment were combined, the result was more significant than the cervical joint treatment group.

What really matters for patients with headaches is not only pain, but quality of life. In this study, the HIT-6 was used to evaluate whether therapeutic interventions actually significantly improved the quality of life for patients with headaches [32].

As a result of this study, after the second treatment in the temporomandibular joint treatment group, improvement was achieved by 48.36 points with no or little headache effect (36-49 points).

Patients with tension headaches often complain of pain in the temporal and mandibular joint as well as pain around the skull, neck, and face and ears [10]. Because the temporomandibular joint treatment applied in the temporomandibular joint treatment group improved the tender points of the masticating-related muscles that were not resolved in the cervical manual therapy treatment, it can be considered that the tension of the masticatory muscle was additionally relieved among the muscles that caused tension headaches [33]. Therefore, it is thought that the temporomandibular joint treatment could further improve the quality of life than the cervical manual therapy group by solving the constant pressure and tightness around the tempor- 
omandibular joint.

Since the NDI can evaluate cervical function and is related to headaches, this study was used to evaluate neck function in patients with chronic tension headaches [34].

In the preceding study, 43 patients with headaches were divided into the temporomandibular joint treatment group and the conservative treatment group [15]. As a result of the study, neck function was significantly improved in the temporomandibular joint treatment group $(p<0.05)$, and there was a significant difference in retest after 6 months $(p<0.05)$. In this study, the temporomandibular joint treatment group showed significant improvement in neck function when compared to other groups $(p<0.05)$. These results can be considered to support the evidence that temporomandibular joints and cervical joints provide a mutual influence as shown in previous papers [35]. Therefore, temporomandibular joint treatment is effective for improving neck function as well as headache pain in patients with chronic tension headaches.

The limitation of this study is that it is difficult to generalize because the number of subjects is not large enough, and it is difficult to confirm the long-term effect of the intervention because a post-test analysis was not performed. In future studies, a large sample should be recruited and studies should be conducted to confirm the long-term application effects.

In this study, there was a significant improvement when using cervical manual therapy, temporomandibular joint therapy in combination with cervical manual therapy, and temporomandibular joint therapy in patients with chronic tension headache related to pericranial tenderness, pain, quality of life, and neck function were compared with the group that applied only manual therapy and the group that applied only conservative physical therapy. When establishing a treatment plan for patients with chronic tension headaches, not only is it important to manipulate the muscles around the neck but also important to consider the management of the muscles involved in the movement of the temporomandibular joint.

\section{Acknowledgements}

This paper was supported by the Sahmyook University.

\section{Conflict of Interest}

The authors declared no potential conflicts of interest with respect to the research, authorship, and/or publication of this article.

\section{References}

1. Diamond S, Bigal ME, Silberstein S, Loder E, Reed M, Lipton RB. Patterns of diagnosis and acute and preventive treatment for migraine in the United States: results from the American Migraine Prevalence and Prevention study. Headache 2007;47: 355-63.

2. Steiner TJ, Birbeck GL, Jensen RH, Katsarava Z, Stovner LJ, Martelletti P. Headache disorders are third cause of disability worldwide. J Headache Pain 2015; 16:58.

3. Jennum P, Jensen R. Sleep and headache. Sleep Med Rev 2002; 6:471-9.

4. Levin M. The International Classification of Headache Disorders, 3rd edition (ICHD III) - changes and challenges. Headache 2013;53:1383-95.

5. Waldie KE, Buckley J, Bull PN, Poulton R. Tension-type headache: a life-course review. J Headache Pain Manag 2015;1:2.

6. Mathew NT. The prophylactic treatment of chronic daily headache. Headache 2006;46:1552-64.

7. Fernández-de-las-Peñas $\mathrm{C}$, Cuadrado $\mathrm{ML}$, Arendt-Nielsen L, Simons DG, Pareja JA. Myofascial trigger points and sensitization: an updated pain model for tension-type headache. Cephalalgia 2007;27:383-93.

8. Natoli JL, Manack A, Dean B, Butler Q, Turkel CC, Stovner L, et al. Global prevalence of chronic migraine: a systematic review. Cephalalgia 2010;30:599-609.

9. Lee HK, Hong JP, Chun YH. Orofacial evaluation of tension-type headache in dentistry. J Oral Med Pain 2004;29:395406.

10. Costa AL, D'Abreu A, Cendes F. Temporomandibular joint internal derangement: association with headache, joint effusion, bruxism, and joint pain. J Contemp Dent Pract 2008;9:9-16.

11. Glaros AG, Urban D, Locke J. Headache and temporomandibular disorders: evidence for diagnostic and behavioural overlap. Cephalalgia 2007;27:542-9.

12. Ayesh EE, Jensen TS, Svensson P. Hypersensitivity to mechanical and intra-articular electrical stimuli in persons with painful temporomandibular joints. J Dent Res 2007;86:1187-92.

13. Mongini F. Temporomandibular disorders and tension-type headache. Curr Pain Headache Rep 2007;11:465-70.

14. Rocha CP, Croci CS, Caria PH. Is there relationship between temporomandibular disorders and head and cervical posture? A systematic review. J Oral Rehabil 2013;40:875-81.

15. von Piekartz H, Lüdtke K. Effect of treatment of temporomandibular disorders (TMD) in patients with cervicogenic headache: a single-blind, randomized controlled study. Cranio 2011;29: 43-56.

16. Von Piekartz HJM. Craniofacial pain: neuromusculoskeletal assessment, treatment and management. Oxford: Elsevier Butterworth-Heinemann; 2007.

17. Saha FJ, Pulla A, Ostermann T, Miller T, Dobos G, Cramer H. Effects of occlusal splint therapy in patients with migraine or tension-type headache and comorbid temporomandibular disorder: a randomized controlled trial. Medicine (Baltimore) 2019;98:e16805. 
18. von Piekartz H, Hall T. Orofacial manual therapy improves cervical movement impairment associated with headache and features of temporomandibular dysfunction: a randomized controlled trial. Man Ther 2013;18:345-50.

19. Kalamir A, Pollard H, Vitiello AL, Bonello R. Manual therapy for temporomandibular disorders: a review of the literature. $\mathrm{J}$ Bodyw Mov Ther 2007;11:84-90.

20. Moraska A, Chandler C. Changes in clinical parameters in patients with tension-type headache following massage therapy: a pilot study. J Man Manip Ther 2008;16:106-12.

21. Jull G, Trott P, Potter H, Zito G, Niere K, Shirley D, et al. A randomized controlled trial of exercise and manipulative therapy for cervicogenic headache. Spine (Phila Pa 1976) 2002;27:183543; discussion 1843 .

22. Kelly AM. The minimum clinically significant difference in visual analogue scale pain score does not differ with severity of pain. Emerg Med J 2001;18:205-7.

23. Bijur PE, Silver W, Gallagher EJ. Reliability of the visual analog scale for measurement of acute pain. Acad Emerg Med 2001;8: 1153-7.

24. Chu MK, Im HJ, Ju YS, Yu KH, Ma HI, Kim YJ, et al. Validity and reliability assessment of Korean Headache Impact Test-6 (HIT-6). J Korean Neurol Assoc 2009;27:1-6.

25. Kosinski M, Bayliss MS, Bjorner JB, Ware JE Jr, Garber WH, Batenhorst A, et al. A six-item short-form survey for measuring headache impact: the HIT-6. Qual Life Res 2003;12:963-74.

26. Trouli MN, Vernon HT, Kakavelakis KN, Antonopoulou MD, Paganas AN, Lionis CD. Translation of the Neck Disability Index and validation of the Greek version in a sample of neck pain patients. BMC Musculoskelet Disord 2008;9:106.

27. Lee H, Nicholson LL, Adams RD, Maher CG, Halaki M, Bae SS. Development and psychometric testing of Korean language ver- sions of 4 neck pain and disability questionnaires. Spine (Phila Pa 1976) 2006;31:1841-5.

28. Castien RF, van der Windt DA, Grooten A, Dekker J. Effectiveness of manual therapy for chronic tension-type headache: a pragmatic, randomised, clinical trial. Cephalalgia 2011; 31:133-43.

29. Castien RF, van der Windt DA, Dekker J, Mutsaers B, Grooten A. Effectiveness of manual therapy compared to usual care by the general practitioner for chronic tension-type headache: design of a randomised clinical trial. BMC Musculoskelet Disord 2009;10:21.

30. Gonçalves DA, Speciali JG, Jales LC, Camparis CM, Bigal ME. Temporomandibular symptoms, migraine, and chronic daily headaches in the population. Neurology 2009;73:645-6.

31. Olivo SA, Bravo J, Magee DJ, Thie NM, Major PW, Flores-Mir C. The association between head and cervical posture and temporomandibular disorders: a systematic review. J Orofac Pain 2006;20:9-23.

32. Stovner Lj, Hagen K, Jensen R, Katsarava Z, Lipton R, Scher A, et al. The global burden of headache: a documentation of headache prevalence and disability worldwide. Cephalalgia 2007;27: 193-210.

33. Fernández-de-Las-Peñas C, Alonso-Blanco C, Cuadrado ML, Miangolarra JC, Barriga FJ, Pareja JA. Are manual therapies effective in reducing pain from tension-type headache?: a systematic review. Clin J Pain 2006;22:278-85.

34. Posadzki P, Ernst E. Spinal manipulations for tension-type headaches: a systematic review of randomized controlled trials. Complement Ther Med 2012;20:232-9.

35. Eriksson PO, Häggman-Henrikson B, Nordh E, Zafar H. Co-ordinated mandibular and head-neck movements during rhythmic jaw activities in man. J Dent Res 2000;79:1378-84. 